\title{
Mitigating Air Delay: An analysis of the Collaborative Trajectory Options Program
}

\author{
Daniel Alberto Pamplona \\ Air Transportation Department \\ Aeronautics Institute of Technology \\ Sao Jose dos Campos, Brazil \\ pamplonadefesa@gmail.com
}

\author{
Claudio Jorge Pinto Alves \\ Air Transportation Department \\ Aeronautics Institute of Technology \\ Sao Jose dos Campos, Brazil \\ claudioj@ita.br
}

\begin{abstract}
Congestion is a problem at major airports in the world. Airports, especially high-traffic ones, tend to be the bottleneck in the air traffic control system. The problem that arises for the airspace planner is how to mitigate air congestion and its consequent delay, which causes increased cost for airlines and discomfort for passengers. Most congestion problems are fixed on the day of operations in a tactically manner using operational enhancements measures. Collaborative Trajectory Options Program (CTOP) aims to improve air traffic management (ATM) considering National Airspace System (NAS) users business goals, particularities faced by each flight and airspace restrictions, making this process more flexible and financially stable for those involved. In CTOP, airlines share their route preferences with the air control authority, combining delay and reroute. When CTOP is created, each airline might decide its strategy without knowledge of other airline's flights. Current solutions for this problem are based on greedy methods and game theory. There is potential space to improve. This paper examines CTOP and identifies important strategic changes to ATM adopting this philosophy, particularly in Brazil.
\end{abstract}

Keywords-CTOP; collaborative trajectory options; air traffic management; ATM

\section{INTRODUCTION}

Air delay is an existing problem in most airports around the world, bringing higher cost to the airlines and discomfort to the passengers. This type of inefficiency brings economic consequences for all stakeholders involved in the airline business. Authors in [1] reported that flights in Europe overdue to airspace inefficiencies and capacity bottlenecks are delayed 10 minutes on average per flight. It is estimated that, on average, consumer benefits per flight due to airspace modernization will count for $€ 32$ with higher levels for business passengers. In the next 20 years the projected demand for civil aviation market varies from 32,600 [2] new aircrafts (freighter and passenger) to 38,050 airplanes [3]. Single-aisle airplanes are expected to command the largest share of the new deliveries, with an estimated need of 26,730 airplanes [2]. The total fleet is expected to double in 15 years. In this context, some cities are expected to concentrate the air demand with long-haul and regional traffic, creating global hubs. The air traffic growth is concentrated in a few cities. In Latin America, since $2007,45 \%$ of the traffic growth is accounted by just 10

Corresponding author: Daniel Alberto Pamplona airports. These airports are not just transport hub exchanges but they arise as the cornerstones of new urban and economic global centers [4].

Delay is one of the consequences of this flight concentration and is a constant problem in most big airports. In 2014, the average delay per delayed (ADD) flight in Europe was 26 minutes. In 2013, 7.9\% of all flights in Brazil were delayed more than 30 minutes, and $3.1 \%$ were delayed more than 60 minutes. In 2010, 24\% of all flights in Europe and 18\% of all flights in USA were delayed more than 15 minutes $[5,6]$. More than half of delays are caused by airline factors such as technical problems, baggage delays, and passenger related problems. The second largest portion $(22 \%)$ is due to air traffic flow control management (ATFCM) problems. The third largest portion is related to airport problems $(16 \%)$ and the last portion is related to the weather (9\%) [6]. Due to capacity constraints, there is a growing necessity for changes in the air traffic system to accommodate the increasing traffic demand. The fundamental shift in ATM paradigm will be from clearance-based air traffic control (ATC) to trajectory-based ATC operations. This new type of trajectory will include new constraints, for example target time of arrival (TTA), that will improve its predictability and as consequence, and facilitate air traffic controllers' work. There are differences in the capacity constraints for the USA and Europe. For US, the major capacity constraints are founded at major airports and in the terminal airspace around them. In the other hand, in Europe the en route airspace presents capacity constraints [7]. According to [8] there are four performance objectives: (a) Airspace design for more capacity, with an increase of $73 \%$ in 2020 , when compared to the 2005 panorama. In the long term, there will be three times more air space capacity; (b) three times improvement in air safety for 2020 and a ten times increase in the longer term; (c) decrease of $10 \%$ on the environmental impact per flight due to ATM; (d) decrease of $50 \%$ to ATM costs per flight. As [9] reminds, ATM is foremost about safety.

\section{A COOPERATIVE ENVIRONMENT BETWEEN AIRLINES AND AIR TRAFFIC AUTHORITIES}

In 2003, during the 11th Air Navigation Conference, it was agreed upon ICAO members that it was necessary to evolve towards a more collaborative environment. Key to this philosophy is the notion of global information utilization, 
management and interchange. This new philosophy aims to evolve to a holistic, cooperative and collaborative decisionmaking environment. Despite the differences between the members, the actions are balanced to achieve equity and access. The following members comprise the ATM community: (a) airport community, (b) airspace providers, (c) airspace users, (d) ATM service providers, (e) ATM support industry, (f) International Civil Aviation Organization (ICAO), (g) regulatory authorities, and $(\mathrm{h})$ States. In this context of collaboration the collaborative air traffic management (CATM) arises [10].

\section{COLLABORATIVE AIR TRAFFIC MANAGEMENT}

Collaborative ATM (CATM) is an attempt to accommodate aircraft operator preferences to the maximum extent possible with restrictions imposed only when an actual operational need exists. CATM tries to adjust the ATC system to meet real-time demands. The main objective is to give the aircraft operator the opportunity to participate in the decisions rather than the ATC Authority arbitrary defining the restrictions. This means that all airspace operators can work together and collaborate on the decision making [11]. The first implementation of CATM is the collaborative decision-making (CDM).

\section{A. Collaborative Decision-Making}

CDM began in US in 1993 when FAA and major air space users started a cooperative environment. Before 1993, FAA used flight schedules published in the Official Airline Guide (OAG) to forecast preliminary air traffic demand prior to operator's route request. The milestone of CDM was when the industry agreed to share its information, providing real-time, day-of-operations schedules [12]. The notion was that both the service provider (FAA) and the system users (airlines) could benefit from cooperation [13]. It was 1995 when CDM was officially launch in the US, when FAA and the industry group defined roles and responsibilities and the foundation for a collaborative air traffic management system was laid [12]. $\mathrm{CDM}$ is structured in the following manner: the basis of the CDM process is: (a) common situational awareness: where all parties must know the constraints, with a shared view of the constraints in the system. Above is the (b) distributed planning: where all parties must be able to react to the constraints in a manner where decisions are made at the most appropriate point. In the top is the (c) analytical capability: where all parties must measure what happened in order to improve the system and is the pillar of the collaborative paradigm [13]. In Europe, CDM was implemented in early 2000's as Airport CDM (A-CDM), because virtually all European airports have slot controls and scheduled operations generally are within airport capacities [14, 15]. Today, CDM is well developed in Europe and USA [16]. Allied with this collaborative environment, air traffic flow management (ATFM) programs were created to reduce the scale and cost during times of adverse weather and heavy traffic demand [15].

\section{B. Air Traffic Flow Management (ATFM)}

ATFM is a function of ATM established with the objective of contributing to a safe, orderly and expeditious flow of traffic while minimizing delays. The purpose of ATFM is to balance air traffic demand with airspace and/or airport capacity to ensure the most efficient use of the airspace system [17]. To achieve those objectives of optimum flow traffic, the following measures include, but are not limited to: a) allocating and updating departure slots, (b) allocating and updating arrival slots, (c) allocating and updating en route slots, (d) re-routing of traffic, (e) alternate flight profiles, (f) minutes-in-trail assignments, (g) mile-in-trail assignments, (h) airborne holding, and (i) ground-holding [17]. ATFM programs developed to handle problems in the en route airspace have been quite successful in mitigating the cost of disruptions, although their success has been limited due to inflexibilities in incorporating flight operator's specific needs and adapting to changing weather and traffic conditions [18]. Recently, the NextGen and SESAR programs are looking for a shift in the ATC method moving for trajectory-based operations (TBO). Linked to this, FAA has recently implemented a new ATFM program, CTOP $[18,19]$.

\section{TRAJECTORY-BASED OPERATIONS}

A trajectory can be defined as the four-dimensional flight path of an aircraft through space and time (4D). The TBO concept means a move from base method ATC to a trajectorybased system of ATM. In this new concept, the aircraft will be assigned flexible and negotiated trajectories and the ATC will have to manage those routes, with the air traffic controllers performing a strategic traffic flow coordinator. This will allow maximum utilization of available airspace and providing advanced navigational capabilities for those aircrafts flying for example RNP trajectories [11]. For operating in this new concept, these will be necessary: (a) the aircraft will be required to transmit and receive aircraft and navigational data in a precise manner, (b) new surveillance equipment, (c) improved aircraft avionics capabilities, (d) advanced automation systems, and (e) automated conflict probes. Enabling TBO requires interactive and integrated decisions and control actions spanning each time horizon to include capacity management, flow contingency management and trajectory management. A critical requirement is the air navigation service provider (ANSP) enabling the stakeholder access and common awareness of the air traffic system capacity and constraints, in the present and future (predicted) situation. CTOP provides through trajectory option set (TOS) an initial foundation of the TBO [20]. TBO is the new ATC concept that moves from a base method to a trajectory-based method. Collaborative Trajectory Options Program (CTOP) is one of the ATM initiatives and it is associated with the idea of a constrained area. Inside the CTOP, there is the Trajectory Options Set (TOS) a set of trajectories that are chosen by the airlines in a constrained area and the Four-Dimension Trajectory (4DT) that is a flight path of an aircraft through space (three-dimension) and time (one-dimension) [21].

\section{A. Collaborative Trajectory Options Program}

CTOP relates to the idea of a constrained area. CTOP is one of many new traffic management initiatives been developed within CATMT and is a part of the NextGen and SESAR initiatives. CTOP is a method of managing demand through constrained airspace. In CTOP, customers are allowed to communicate their preferences in a TOS. The customers can choose between route and delay [19]. CTOP is used anywhere 
there is a constraint in the air traffic system. The most common constraints are weather and air traffic volume. The CTOP program provides greater flexibility for the airspace planner in managing capacity by allowing ground delays and re-routes to be considered together. According to [18] CTOP has similarities with the previous en route ATFM programs with the difference that it considers flight operator's submitted en route resources preferences.

\section{B. Trajectory Options Set}

A TOS will allow the airlines to manage a flight by telling the ATC the route and delay options that the clients are willing to accept. The TOS may contain multiple trajectories options, with different routes, altitude or speed per trajectory. The difference between a flight plan and a TOS is that the flight plan contains a single request with a defined route, altitude and speed. TOS may contain multiple trajectory options, with each one of the options, containing routes, altitudes or speeds [19]. In the current air traffic control system (ATCS) the pilot determines through a flight plan the flight's objective (destination airport) and how to reach it, deciding which route is best, the proposed altitude, the cruising airspeed, the time of departure, climb and descent profiles. To control an airplane while flying, the pilot can be qustioned by the air traffic controller if the parameters requested in the flight plan form are maintained or can determine the aircraft's flight profile by interpreting the flight track, azimuth and altitude information displayed on the radar scope [11]. In the current ATC configuration, the system aims to satisfy each pilot's request for a specific route or altitude. It may be necessary to apply procedural restrictions to ensure positive aircraft separation. The constant use of air space restrictions results in increased fuel use, increased flight times, loss of flexibility, and, occasionally, reduced traffic flow. In the other hand, great care must also be taken to not overload the air controller. The routine imposition of procedural restrictions reduces the controller's workload, and consequently decreases the potential loss of separation between aircrafts and decreases the number of planes flying in an area. These procedural restrictions tend to keep an aircraft at inefficient altitudes. Since the constrained aspect is the controller's capacity to coordinate clearances and predict separation conflicts, and not airspace saturation, an automated process would reduce the need for rigid procedural restrictions on system capacity. In this aspect, manual air traffic control procedures need to be improved with computer-based decision support systems for the ATC to become more efficient and capable. The aircraft separation is nowadays human dependent, maintained by air traffic controllers who use radar screens to visualize aircraft flight paths, make subjective judgements as future aircraft positions and potential conflicts, and mentally develop alternate flight paths [11].

The operators must express their preferences among different flight options, which must be expressed in terms of a relative trajectory cost (RTC). Each option will be evaluated based on customer's preference expressed by the RTC [19]. The RTC of a flight option is an expression of the number of minutes of delay that would have to be imposed upon the operator's most preferred trajectory option before some other flight option becomes a desirable alternative. Upon submitting a TOS, the CTOP and CACR algorithms assign routes and/or ground delay to flights by attempting to provide the operator a minimum adjusted cost. The minimum adjusted cost is the sum of the delay assigned to a flight plus its RTC, while ensuring that traffic is limited within the program flow constrained area (FCA) to a specified capacity [20]. The fundamental principle behind CTOP and TOS is the four-dimension trajectory.

\section{Four-Dimension Trajectory}

Four-dimension trajectory (4DT) is the pillar of the new ATM, whereby time-based operations progress to trajectorybased operations and in the long term achieve performancebased operations. A 4DT is defined as a precise description of an aircraft path in space and time. Waypoints are used to represent specific steps along the path, which is earthreferenced with a proper latitude and longitude [7]. What distinguishes a 4DT is that the path contains altitude description for each waypoint and indications about the time at which the trajectory will be executed. Some waypoints in the 4DT path may be associated with controlled time of arrival (CTA) or required time of arrival (RTA). In a CTA, it may be assigned a target time of arrival (TTA). The aircraft must meet this TTA requirement within a specified time of tolerance. The CTA represents a time window for the aircraft to pass through a specific waypoint. It is normally used to regulate traffic flows entering congested en route/arrival/departure airspace. The main idea is to establish a sequence of spatial and temporal windows. This sequence will represent milestones to meet during flight execution [22]. To achieve the desired RTA, aircraft's speed must be adjusted and regulated along the trajectory to arrive at a specific waypoint at a specified time, improving the predictability of the aircraft-flying path. The problem is that the time of arrival over a fixed point is not a function of aircraft's airspeed alone, but it depends upon the winds and temperature that the aircraft will encounter in its route [23]. In Europe, in the SESAR program, the 4DT is often called reference business trajectory (RBT). The term reference is used because once a trajectory is chosen, it will become the reference trajectory which the airspace user agrees to fly and all the service providers agree to facilitate with their respective services. This name difference is basically caused by the European consortium which wants a more collaborative environment, where trajectories are agreed between all the ATM stakeholders, for example ATC, airports, airlines, military and general aviation. This 4DT will be executed gateto-gate by the aircraft [7].

SESAR's and NextGen's core concept is to structure ATM around aircraft TBO. To achieve this milestone, it is necessary for the aircraft to achieve the 4DT with accuracy/reliability, to accurately and fast pass information via data link, huge improvements in surveillance capabilities are demanded as well as automation and decision support tool capabilities and huge improvements in computer/equipment processing power and speed [9]. The 4D concept is consistent within ICAO Aviation System Block Upgrade (ASBU) and with ICAO Global Air Navigation Plan and Global Air Traffic Management Operational Concept. Some authors divide 4DT in two phases: initial 4DT (I4DT) and full 4DT (4DT). The objective of an I4DT is to optimize the arrival phase of a flight at an airport. 
To achieve this goal, the airborne and ground trajectories must be synchronized around a common unique reference designated by a $2 \mathrm{D}$ point or metering fix (MF) and a time constraint. The trajectory negotiation process begins when the aircraft is about $200 \mathrm{NM}$ or 40 minutes from its destination. The negotiation is made via a data link between the ATC and the aircraft and includes the standard terminal arrival route (STAR) and approach procedures applicable to the metering fix. The final 4DT is a lateral route with altitude, speed and time constraints over waypoints in the trajectory [24]. For the implementation of the I4DT function onboard, the following avionics systems are necessary: (a) cockpit display systems: it must display relevant data related to the engagement and monitoring of the 4DT, (b) flight management system: the onboard computed prediction and the system performance requirements are consistent, (c) communication system: must be able to manage the ADS-contract and the controller-pilot data link communication (CPDLC) applications. An information management platform is necessary to allow this entire collaborative environment. The system wide information management (SWIM) platform will provide the infrastructure and services necessary to deliver network-enabled information access to a multitude of ATM system users. The system must integrate with a variety of legacy sub-system over many years. SWIN is described as a framework enabling authorized applications and services to reliably and securely share information. SWIN will allow the necessary trajectory functions exchange functions. This will permit a system coordinated 4DT plans [23].

\section{FUTURE SCOPE}

Figure 1 shows the most important metrics for result comparison of the 4DTs in the key performance areas.

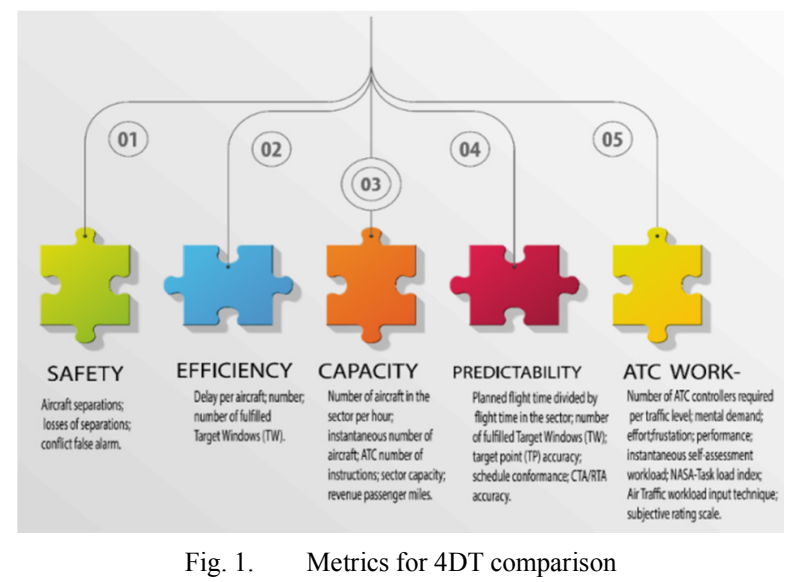

To achieve TBO environment, the following technologies are considered necessary: (a) advanced flight management system (FMS) capabilities: 4DT can only exist with accurate (CTA) capabilities. The key factors that impact system accuracy are wind and temperature data. (b) Data communication: the voice communication channel between ATC and cockpit will not be sufficient to handle the amount of traffic. It will be necessary to introduce data communication that will decrease the controller's workload. One of the key aspects is the balance between the new airspace capacity and the controller task load [7]. ATM system depends critically on the rate that controllers can process aircraft through airspace sectors [9]. (c) ADS-B: this technology will replace the RADAR as surveillance instrument. (d) Air traffic control decision support tools: There is a necessity to implement decision support tools (DST) for air traffic controllers. DST will be necessary to provide air traffic controllers with acceptable levels of workload. DST will have to handle the trajectories predicted for the system, and will allow to share and negotiate 4DT, and keep the traffic separated. They will be able to have the capability of conflict detection and resolution.

The CTOP, which started on March 2014, is a new concept for traffic flow management (TFM). However, some characteristics could be brought to Brazil to be implemented, as a future program. The application of preferential routes to IFR has the objective of optimizing the airspace's use and allowing the better planning of flights. Also, it intends to reach better use of aircraft's RNAV navigation systems to keep air traffic flow and its high safety standards. These preferential routes could be used as initial routes in the TOS since they are the most advantageous for air traffic service (ATS) provider and airline companies. Since these routes represent the optimized routes, they represent for airline companies the fastest routes representing lesser cost, and for ATS provider represent that flights will follow routes which are contained in sectors which could absorb the increase in traffic flow. Another important step towards the CTOP implementation would be the observation of airspace's characteristics, such as certain regions which present degraded weather conditions. One possible application is at The Rio de Janeiro and Sao Paulo air Terminals, as shown in Figure 2. This is the region with the largest aircraft movement in Brazil. Due to its geographical proximity and air movement growth trend, it is estimated that in the coming decades, there will be an increase in flight delay.

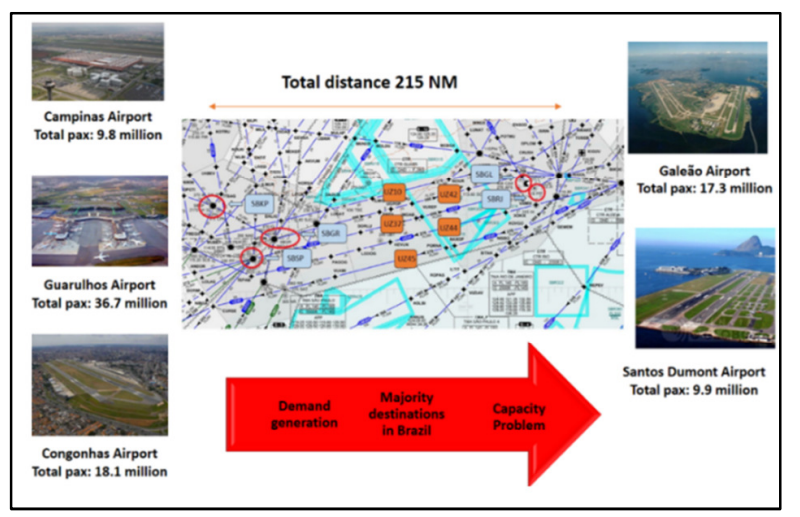

Fig. 2. Possible implementation in Brazil

This could be done through the assessment of meteorological maps and through the experience of air traffic controllers and other workers in the sector. This analysis would allow identifying the most common constrained areas in the Brazilian airspace and it would be an initial step to create the alternative routes to be part of the TOS. Then, the use of fasttime simulation could allow identifying the cost of each 
trajectory relative to one another. Cost parameters such as travelling time and fuel burn could be evaluated and they would compound the relative cost for each trajectory. Validating the trajectories with all stakeholders is an important step to ensure that TOS satisfies their needs.

\section{CONCLUSION}

In 15 years, the total commercial fleet is expected to double and some cities are expected to concentrate air demand with long-hauls and regional traffic creating global hubs. Delay is one of the consequences of this flight concentration and due to capacity constraints there is a growing necessity for changes in the air traffic system to accommodate the increased traffic demand. Air traffic flow management aims to balance traffic demand with airspace. The purpose of ATFM is to balance air traffic demand with airspace and/or airport capacity to ensure the most efficient use of the airspace system. A fundamental change will be from clearance-based ATC to trajectory-based ATC operations and the implementation of the CTOP. CTOP aims to improve air traffic management considering National Airspace system users business goals, particularities faced by each flight, and airspace restrictions, making this process more flexible and financially stable. TBO will be a fundamental pillar in this new operational scenario and will permit more and extreme use of accessible airspace. With its increasing demand for air transportation, Brazil, especially Rio de Janeiro and Sao Paulo region, is a serious candidate for the implementation of such technologies. This district is the one with the biggest airplane deployment in Brazil. Because of its land closeness and air deployment pattern, it is assessed that in the coming decades, there will be an expansion in flight delay. Besides air saturation, the full operational implementation of new technologies is also necessary. Research is undergoing to embed all the needed capabilities in SESAR and NextGen operational environment.

\section{REFERENCES}

[1] G. Burghouwt, R. Lieshout, T. Boonekamp, V. van Spijker, Economic Benefits of European Aairspace Modernization, SEO Amsterdam Economics, 2016

[2] Airbus, Global Market Forecast 2014-2034, 2015

[3] Boeing, Current Market Outlook 2015-2034, 2015

[4] V. Bamberger, M. Blondel, Mega-Aviation Cities' Project, Arthour D. Little, 2013

[5] Brazilian National Civil Aviation Agency (ANAC), Air Transport Yearbook, 2014

[6] European Organisation for the Safety of Air Navigation (EUROCONTROL), Network Operations Report for 2014, 2015

[7] G. Enea, M. Porretta, "A comparison of 4D-trajectory operations envisioned for Nextgen and SESAR, some preliminary findings", 28th Congress of the International Council of the Aeronautical Sciences, Brisbane, Australia, September, 23-28, 2012

[8] SESAR Consortium, Milestone Deliverable D3: The ATM Target Concept, 2007

[9] P. Brooker, "Sesar's ATM Target Concept: Keys to sucess", available at: https://dspace.lib.cranfield.ac.uk/handle/1826/2941, 2008

[10] International Civil Aviation Organization (ICAO), Doc 9854 Global Air Traffic Management Operational Concept, 2005

[11] M. Nolan, Fundamentals of Air Traffic Control, Cengage Learning, 2011
[12] Transportation Research Board (TRB), Guidebook for Advancing Collaborative Decision Making (CDM) at Airports, 2015

[13] M. C. Wambsganss, "Collaborative decision making in air traffic management", in: New Concepts and Methods in Air Traffic Management, pp. 1-15, Springer, 2001

[14] M. O. Ball, "Collaborative Decision Making: US vs Europe", 2015 NEXTOR Workshop on Global Challenges to Improve Air Navigation Performance, Asilomar, USA, February 11-13, 2015

[15] European Organisation for the Safety of Air Navigation (EUROCONTROL), Airport CDM Implementation - The Manual for Collaborative Decision Making, 2012

[16] M. O. Ball, R. Hoffman, A. Mukherjee, "Ground delay program planning under uncertainty based on the ration-by-distance principle", Transportation Science, Vol. 44, pp. 1-14, 2010

[17] International Civil Aviation Organization (ICAO), Doc 9971 Manual on Collaborative Air Traffic Flow Management, 2014

[18] A. Kim, M. Hansen, "Some insights into a sequential resource allocation mechanism for en route air traffic management", Transportation Research Part B: Methodological, Vol. 79, pp. 1-15, 2015

[19] Federal Aviation Administration (FAA), AC 90-15 - Collaborative Trajectory Options Program (CTOP): Document Information, 2014

[20] A. A. Aslinger, L. Martin, W. S. Leber, M. A. Hopkins, "Enabling a modernized NAS ATM infrastructure in support of trajectory based operations", 2012 Integrated Communications, Navigation and Surveillance Conference, Herndon, USA, April, 24-26, 2012

[21] B. Vaaben, J. Larsen, "Mitigation of airspace congestion impact on airline networks", Journal of Air Transport Management, Vol. 47, pp. 54-65, 2015

[22] P. Brooker, "A 4D ATM trajectory concept integrating GNSS and FMS?", The Journal of Navigation, Vol. 67, pp. 617-631, 2014

[23] J. Klooster, K. Wichman, O. Bleeker, "4D trajectory and time-of-arrival control to enable continuous descent arrivals", AIAA Guidance, Navigation and Control Conference and Exhibit, Honolulu, USA, August 18-21, 2008

[24] L. H. Mutuel, P. Neri, E. Paricaud, "Initial 4d trajectory management concept evaluation", Tenth USA/Europe Air Traffic Management Research and Development Seminar, Chicago, USA, June 10-13, 2013 